\title{
MODELLO DELL'ATOMO, TEORIA QUANTISTICA E TAVOLA PERIODICA DEGLI ELEMENTI
}

\author{
GIOVANNI MARIA PROSPERI (*)
}

SunTO. - In questo contributo ho tentato di delineare l'itinerario che ha portato dalla tavola degli elementi, nata in un contesto fondamentalmente chimico, ad una sua interpretazione nell'ambito della Fisica Atomica che coinvolge $\mathrm{i}$ formalismi più avanzati della Fisica Teorica; la Meccanica Quantistica e in ultima analisi anche la stessa Teoria Quantistica dei Campi. Come la tavola è stata insieme alla spettroscopia tra le principali problematiche che hanno stimolato e anche indirizzato la ricerca in questi ultimi ambiti.

$$
* * *
$$

ABSTRACT. - In this paper I attempt to sketch the path that brought from the Table of the Elements, essentially born in an chemical context, to its explanation in the framework of Atomic Physics.Thist involves the most advanced formalisms of Theoretical Physics, Quantum Mechanics and even Quantum Field Theory. I try to stress that the Table together with Spectroscopy has been among the main problems that have stimulated and even addressed the research in the latter frameworks.

\section{INTRODUZIONE}

La Tavola degli Elementi proposta per la prima volta in una forma simile a quella da noi oggi utilizzata dal chimico russo D. Mendeleev nel 1869 e, indipendentemente, qualche mese dopo dal chimico tedesco L. Meyer, rappresenta certamente innanzitutto una pietra miliare nello sviluppo della Chimica, permettendo di dare un ordine al quadro delle sostanze che progressivamente venivano scoperte e aprendo ancora una volta ad una prospettiva di semplicità e valore estetico nelle leggi della natura.

(*) Istituto Lombardo Accademia di Scienze e Lettere, Milano, Università degli Studi di Milano, Italia. E-mail: Giovanni.Prosperi@mi.infn.it 
Una comprensione del suo significato profondo si è però potuto avere solo con lo sviluppo della Fisica Atomica a partire dal primo modello di Rutherford nel 1911, dai primi tentativi successivamente di costruire una Teoria Quantistica e infine, verso la metà degli anni venti del '900, dalla formulazione di una Meccanica Quantistica coerente e organica. Insieme soprattutto alla Spettroscopia, una sua comprensione ha posto importanti problemi alla ricerca in questi ultimi ambiti e ha svolto un'azione di stimolo che ha portato, tra l'altro, alla formulazione da parte di W. Pauli prima del principio di esclusione e poi del postulato di antisimmetria per al funzione d'onda elettronica e quindi allo sviluppo delle statistiche quantistiche e del Teorema di connessione tra Spin e Statistica.

In questo contributo voglio appunto cercare di delineare a grandi linee questo percorso. Nella sez. 2 tento di richiamare molto sommariamente i criteri che hanno portato alla formazione della Tavola; nella sez. 3 delineare il ruolo della Fisica atomica e della primitiva Teoria di Bohr Sommerfeld che attraverso il principio di esclusione ha portato a una, sia pure incompleta, sua prima giustificazione; nella sez. 4 cerco di illustrare quello della nuova Meccanica Quantistica non relativistica (come sviluppata soprattutto per opera di L. De Broglie e E. Schroedinger da una parte e di W. Heisenberg, M. Born e P. Jordan dall'altra) nel dare coerenza e nel completare le acquisizioni della vecchia teoria; infine nella sez. 5 voglio appena accennare al contributo ulteriore della Teoria Quantistica dei Campi coni il menzionato teorema di connessione.

\section{La Tavola Periodica}

Convenzionalmente si suole far nascere la Fisica nel senso moderno del termine con Galilei. La Chimica, nonostante la sua lunga preistoria, si può dire nasca, come scienza sperimentale quantitativa, solo oltre un secolo e mezzo dopo con L. A, Lavoisier.

Importante merito di Lavoisier, oltre naturalmente ad aver stabilito la legge di conservazione della massa, è quello di aver ricuperato la definizione di elemento, data precedentemente da R. Boyle, come di sostanza non ulteriormente scomponibile e su questa linea di aver saputo chiarire la composizione di varie sostanze comuni. E' chiaro che, con tale definizione, l'individuazione degli elementi può solo essere il frutto di ricerca sperimentale e ciò pone fine a tutta una ridda di speculazioni a priori. Cominciano ad essere identificati come primi elementi tutti i metalli allo- 
ra conosciuti, alcune altre sostanze come il carbonio e lo zolfo, alcuni gas come l'ossigeno, l'azoto e l'idrogeno. Inizialmente vengono erroneamente considerati tali anche alcuni ossidi, come la soda, la potassa e la calce, che solo successivamente si riuscirà a scomporre.

Segue la formulazione delle prime leggi quantitative di combinazione degli elementi, per dare ragione delle quali J. Dalton formula il suo modello atomico-molecolare, introducendo tra l'altro il concetto di peso atomico, come rapporto tra il peso dell'atomo dell'elemento considerato e quello dell'atomo più leggero, l'atomo di idrogeno. Il modello è perfezionato da A. Avogadro che, sulla base della sua legge sui gas, risolve molte ambiguità, come quella del numero degli atomi di idrogeno che intervengono nella molecola d'acqua e propone l'esistenza anche per le sostanze semplici di molecole costituite da atomi identici. Sarà poi $\mathrm{S}$. Canizzaro a dare al modello nel 1858 una sistemazione definitiva, permettendo tra l'altro una assegnazione univoca dei pesi atomici.

Con la progressiva individuazione e studio dei vari elementi, ci si rese ben presto conto che questi potevano essere raggruppati sulla base di proprietà simili. Ancora nel $1829 \mathrm{~J}$. W. Doebereiner riconosceva l'esistenza di triadi di affinità come Cloro, Bromo, Iodio o Calcio, Stronzio, Bario. Nel 1862 A. E. B. de Chacortois ha l'idea di ordinare gli elementi conosciuti secondo i pesi atomici e constata il ripresentarsi ciclico di sequenze con proprietà simili e nel 1864 J.A.R. Newlands formula una legge delle ottave.

Nel 1869, finalmente, il chimico russo D. Mendeleev e solo pochi mesi dopo e indipendentemente il tedesco L. Meyer proposero la tavola sistematica che dal primo autore ha poi preso il nome. In Fig. 1 è riportato il primo tentativo appunto di Mendeleev, apparso nella prima edizione dei suoi Fondamenti di Chimica. In essa sono inquadrati, pur con vari punti interrogativi, i 64 elementi allora conosciuti. Nella parte superiore questi sono ordinati in colonna secondo i loro pesi atomici, andando a capo su una colonna parallela quando si incontra una sequenza di elementi con proprietà simili a quelle precedente, in modo da allineare sulla stessa riga elementi con le stesse proprietà. Nella parte inferiore gli elementi con proprietà simili sono invece disposti sulla stessa colonna.

La tavola risulta ovviamente molto incompleta rispetto alle conoscenze attuali. Mancano, in particolare, tutti i gas nobili che saranno scoperti e inquadrati nella stessa solo verso la fine del secolo. E' inoltre evidente l'incertezza e la difficoltà nel trovare una collocazione agli elementi di transizione. 


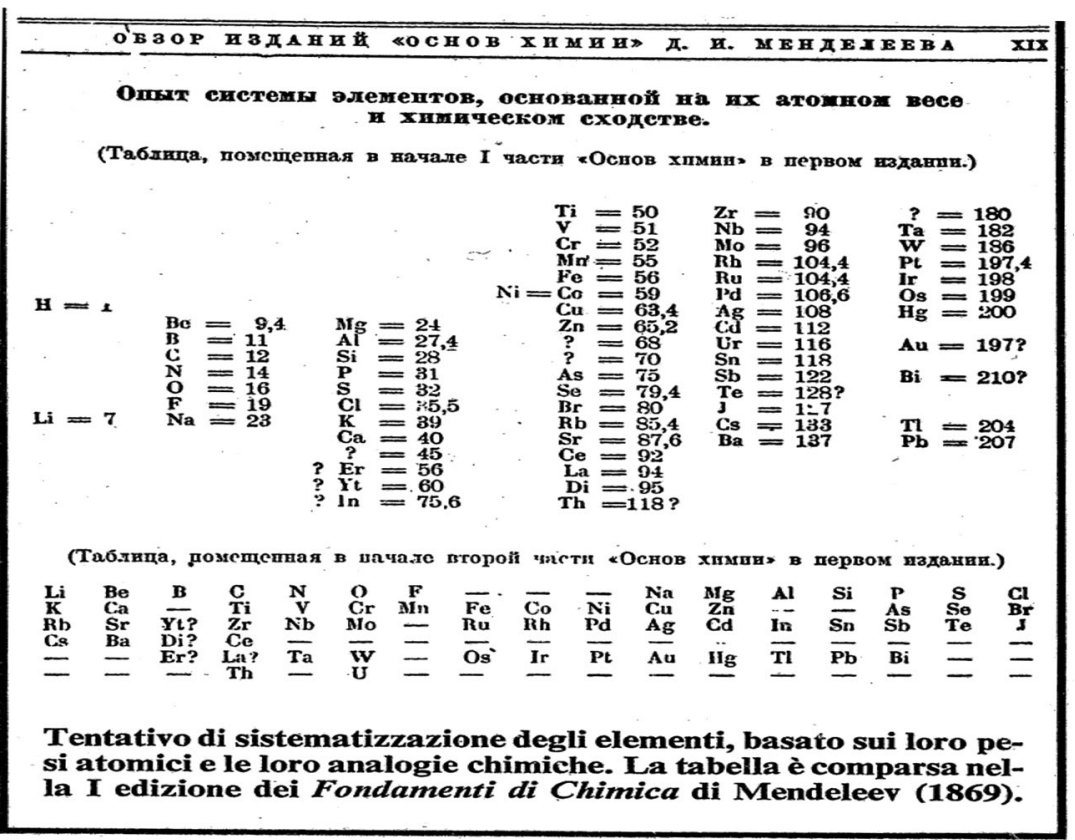

Fig. 1. La prima tavola di Mendeleev (da Enciclopedia Della ScienZa E DELla TeCnicA, voce Chimica inorganica di L. Malatesta,. Mondadori, Milano 1963).

Anche così, per mantenere l'allineamento tra elementi con proprietà simili, Mendeleev fu costretto a lasciare alcuni posti vuoti, che suppose dovessero essere occupati da elementi ancora sconosciuti. Fu così portato a prevedere l'esistenza di un elemento dopo il calcio, lo Scandio, e di due dopo lo Zinco, il Gallio e il Germanio, che furono puntualmente scoperti alcuni anni dopo. Per la stessa ragione fu costretto a invertire l'ordine di successione di altri elementi rispetto al valore del loro peso atomico, rispetto a quello allora conosciuto. A parte errori nella determinazione degli stessi, noi oggi in effetti sappiamo che il corretto ordinamento è quello rispetto al numero atomico $Z$, che si identifica con il numero degli elettroni nell'atomo neutro e non coincide sempre con quello del peso atomico per la varietà della composizione isotopica.

Per aver un criterio univoco per l'assegnazione di $Z$ come indice della posizione di un dato elemento, si dovrà in realtà attendere fino $\mathrm{al}$ 1913, quando Moseley formulerà la sua legge sulla dipendenza della fre- 
quenza di emissione di raggi $\mathrm{X}$ dalla posizione occupata sulla tavola. Mosely osservò che gli spetri a raggi $\mathrm{X}$ di frequenza più elevata di elementi contigui apparivano molto simili, ma con frequenze corrispondenti che si spostavano progressivamente verso i valori maggiori (Fig. 2).

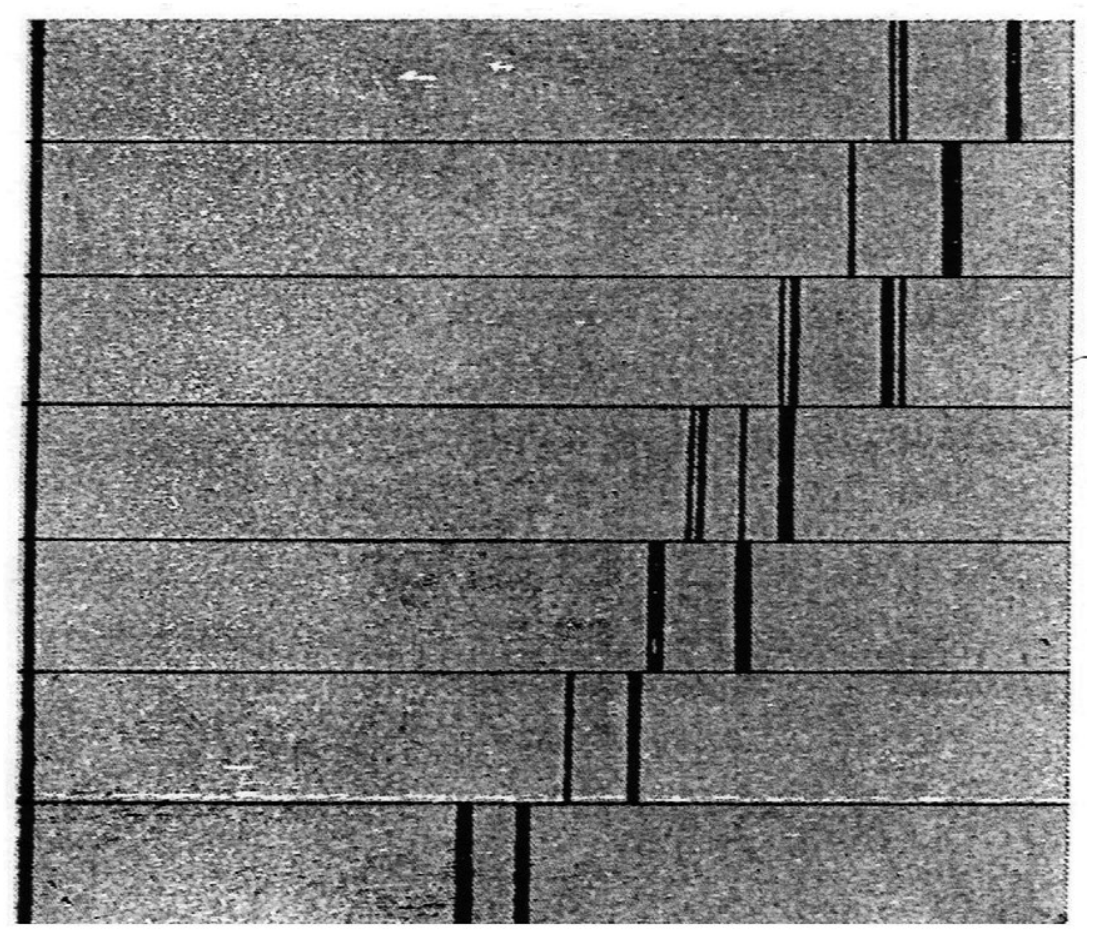

Fig. 2. Lo spettro a raggi $\mathrm{X}$ di alcuni elementi disposti secondo l'ordine che occupano nella tavola (da M. Borm, Fisica Atomica, Boringhieri. Torino 1968).

Precisamente egli stabilì che la radice quadrata della frequenza di ogni determinata riga risulta proporzionale al numero $Z$ (Fig. 3, equivalentemente risulta proporzionale $Z^{2}$ ). E' chiaro che in tal modo ambiguità nell'ordine di due elementi potevano essere immediatamente risolte e l'esistenza di lacune messa in evidenza. Si notino incidentalmente le lacune presenti nei grafici riportati nella Fig. 3, che corrispondono però per lo più semplicemente ad elementi che in condizioni normali sono gassosi, e per i quali ottenere spettri a raggi X è evidentemente difficile. 
In corrispondenza delle successive edizioni del suo libro, Mendeleev aggiornò progressivamente la sua tavola con i nuovi elementi che venivano scoperti. Altre disposizioni furono proposte da altri autori. Fino alla creazione di una Fisica Atomica, tuttavia, la tavola mantenne un carattere puramente empirico. E' solo nell'ambito di quest'ultima e della Teoria Quantistica, che ne costituisce la base, che il suo profondo significato poté essere compreso.

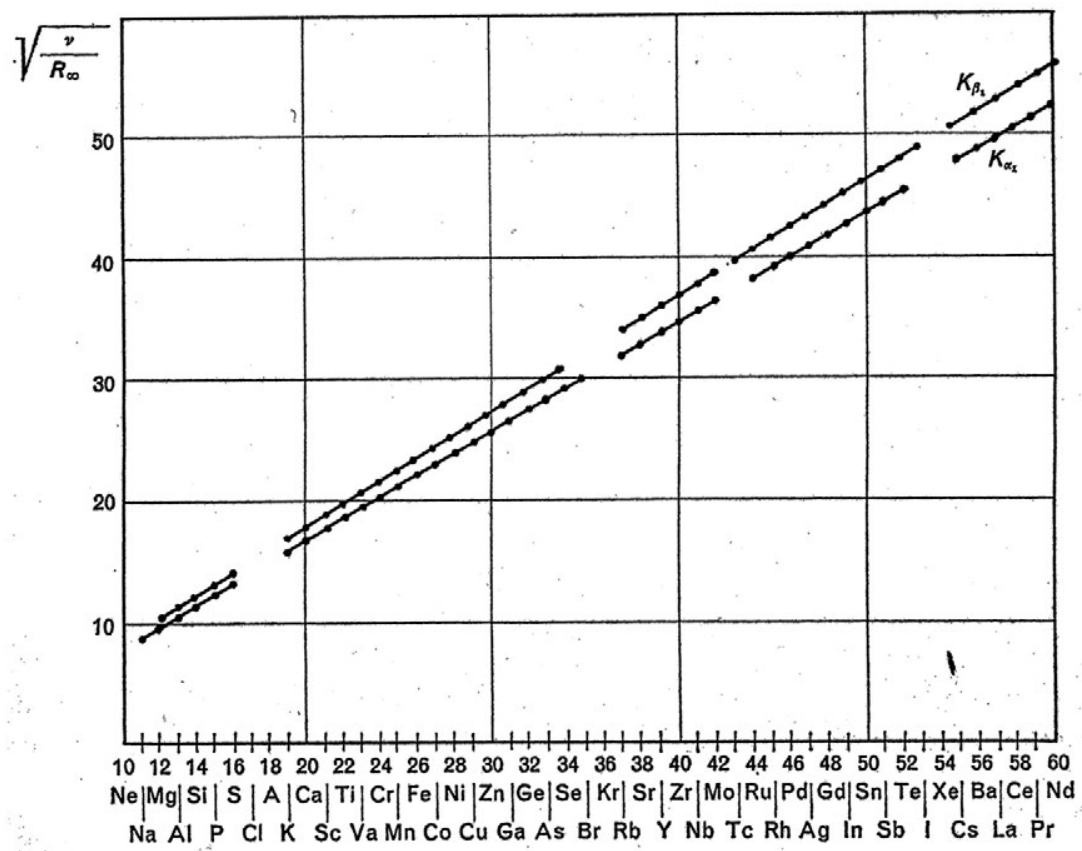

Fig. 3. La legge di Moseley: relazione di proporzionalità tra la radice quadrata della frequenza di due righe corrispondenti dello spettro a raggi $\mathrm{X}$ di vari elementi e il numero $Z$ che specifica la posizione degli stessi nella tavola (da M. Born loc. cit.)

\section{LA NASCITA DELLA Fisica ATOMICA E UNA PRIMA}

GIUSTIFICAZIONE DELLE TAVOLA NEL CONTESTO DELLA

“VECCHIA" TEORIA QuANTISTICA

La Teoria Quantistica nasce con l'ipotesi di M. Planck sul quanto di radiazione.

Nel 1900, per cercare di spiegare le caratteristiche dell'emissione di radiazione elettromagnetica da parte di un corpo solido o liquido 
portato ad una certa temperatura $T$ (problema del corpo nero), Planck si vide costretto ad ammettere che gli scambi di energia tra radiazione e materia potessero avvenire solo per multipli di una quantità discreta detta quanto, proporzionale alla frequenza $v$,

$$
w=h v,
$$

dove $h$ era intesa come una nuova costante universale. Dando ad $h$ un valore opportuno le curve che davano l'intensità della radiazione emessa dalla frequenza e il loro andamento al cambiare della temperatura venivano riprodotte con grande precisione (oggi $h$ è una delle grandezza meglio conosciute, alla terza cifra decimale $h=6,62610^{-34} \mathrm{~J}$ sec) (Fig. 4).

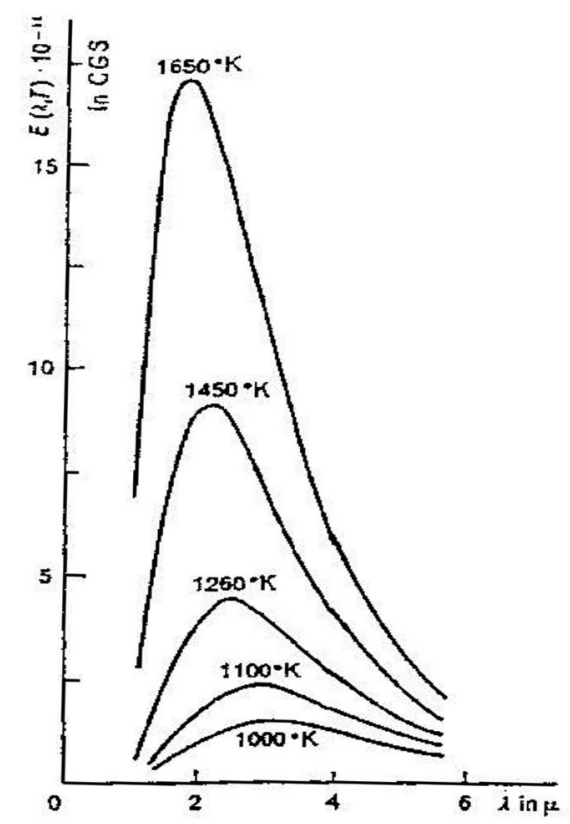

Fig. 4. Curve del potere emissivo del "corpo nero" in funzione della lunghezza d'onda per diversi valori della temperatura $T$. Si tratta delle curve che esprimono la dipendenza dalla lunghezza d'onda della radiazione che si stabilisce all'interno di una cavità le cui pareti sono portate alla temperatura $T$, come può essere osservata attraverso un piccolo foro praticato sulla parete stessa che non ne disturbi apprezzabilmente l'equilibrio. E' questo il modo di osservare il fenomeno di emissione per riscaldamento nella maniera più pura separandolo da altri effetti perturbanti. 
Qualche anno dopo Einstein riprendeva l'idea di Planck, spostando l'attenzione dalla materia, come sostanzialmente aveva fatto questi, alla radiazione e supponeva che quest'ultima si propagasse essa stessa per pacchetti d'onda elementari, portatori di un'energia data dalla relazione di Planck, ma anche, secondo le leggi dell'Elettromagnetismo, di una quantità di moto ottenuta dividendo quest'ultima per la velocità della luce $c$,

$$
p=\frac{w}{c}=\frac{h v}{c} .
$$

$\mathrm{Al}$ quanto di radiazione veniva attribuito in questo modo il carattere di particella e alla radiazione in sostanza una doppia natura, ondulatoria e corpuscolare. Successivamente a questa particella si è dato il nome di fotone.

Una Fisica Atomica con una base sperimentale nasce invece nel 1911. E. Rutherford, per spiegare le caratteristiche della diffusione di un fascio di particelle $\alpha$ provenienti da una sostanza radioattiva da parte della lamina sottile di un metallo pesante, formulò il suo modello di atomo nucleare. Come è noto, secondo tale modello l'atomo si suppone costituito da un nucleo centrale molto piccolo carico positivamente e in cui è concentrata la maggior parte della massa dello stesso e una nuvola di elettroni che gli ruota intorno. Studi successivi con lamine di metalli diversi suggerirono inoltre che la carica del nucleo dovesse essere uguale a $Z e_{0}\left(\operatorname{con} e_{0}=1,6 \cdot 10^{-19} \mathrm{C}\right.$ carica elementare, uguale al valore assoluto della carica dell'elettrone) e quindi $Z$ rappresentasse anche il numero degli elettroni dell'atomo neutro.

Il modello di Rutherford andava però incontro a varie difficoltà. Secondo le leggi dell'elettromagnetismo classico, infatti, una carica elettrica negativa che ruota attorno ad una carica positiva dovrebbe comportarsi come un'antenna ed emettere radiazione di frequenza uguale a quella di rivoluzione attorno a quest'ultima. Un atomo di questo tipo non potrebbe essere stabile. L'elettrone dovrebbe perdere rapidamente energia e ricadere sul nucleo in un tempo molto breve (dell'ordine di $10^{-8}$ secondi). Nel ricadere verso il nucleo, inoltre, la sua velocità angolare dovrebbe progressivamente aumentare secondo la III legge di Keplero e la radiazione essere emessa su tutto uno spettro continuo, esteso dalle frequenze infrarosse fino ai raggi $\mathrm{X}$. Lo spettro di emissione o di assorbimento dei gas allo stato atomico è 
invece uno spettro di righe, costituito da frequenze ben separate organizzate in più serie. In Fig. 5 è riportata la rappresentazione schematica di una delle serie dell'atomo di idrogeno e in Fig. 6 la fotografia di porzioni dello spettro di emissione e di assorbimento di vapori del sodio.

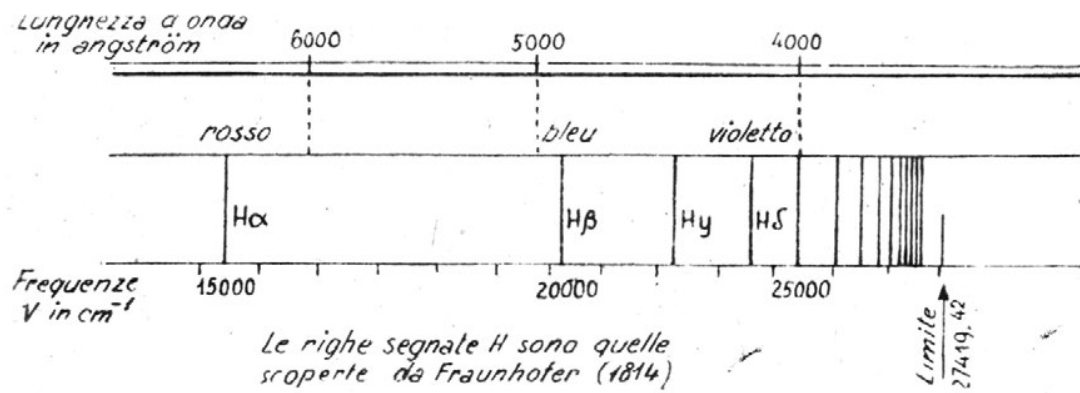

Fig. 5. Lo spettro dell'idrogeno è costituito da più serie di righe che si estendono dall'ultravioletto fino all'estremo infrarosso. In figura è rappresentata schematicamente la serie di Balmer parzialmente nella regione del visibile.

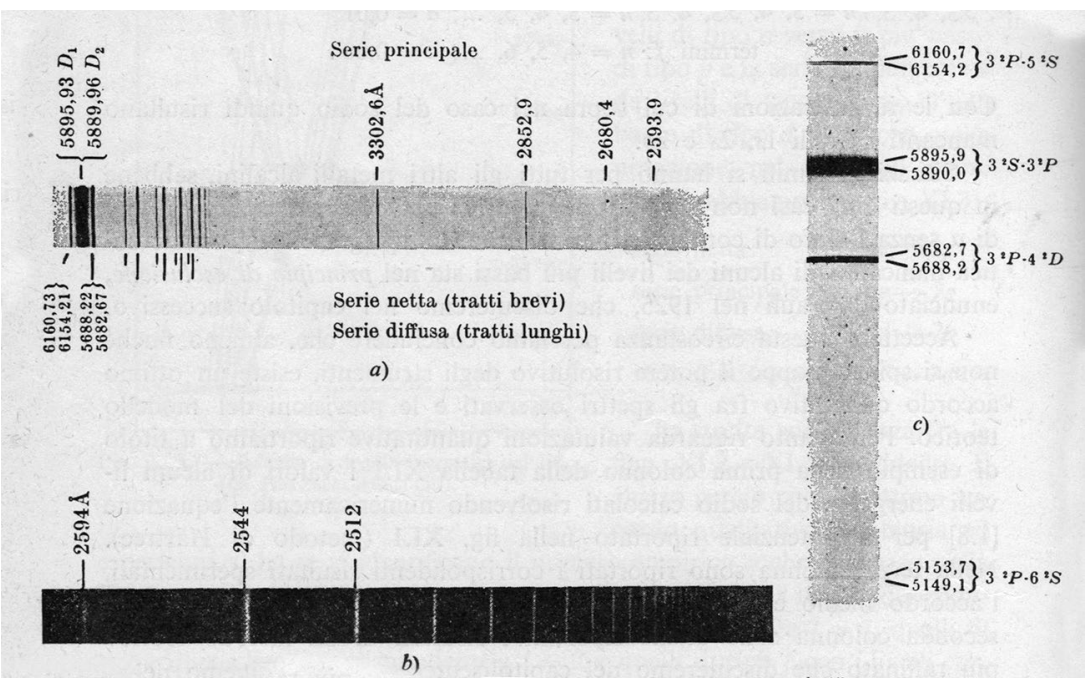

Fig. 6. Porzioni dello spettro di emissione ( $a$ e c) e di assorbimento (b) del sodio. (da G, Herzberg, Spettri atomici e struttura atomica, Boringhieri, Torino, 1957).

Nel 1913 N. Bohr riprese il modello di Rutherford e, per superarne le difficoltà, ammise che, in rottura con le leggi della Meccanica e 
dell'Elettromagnetismo classici, nell'atomo gli elettroni potessero muoversi solo su certe orbite discrete, alle quali quindi competessero valori discreti dell'energia, $E_{1}, E_{2}, \ldots E_{n}, \ldots$ e sulle quali essi non irraggiassero. Suppose che l'irraggiamento dovesse avvenire per transizione da un'orbita di energia superiore $E_{n}$ ad una di energia inferiore $E_{n^{\prime}}$ con emissione (o viceversa nella transizione opposta con assorbimento) di un unico quanto $h v$.

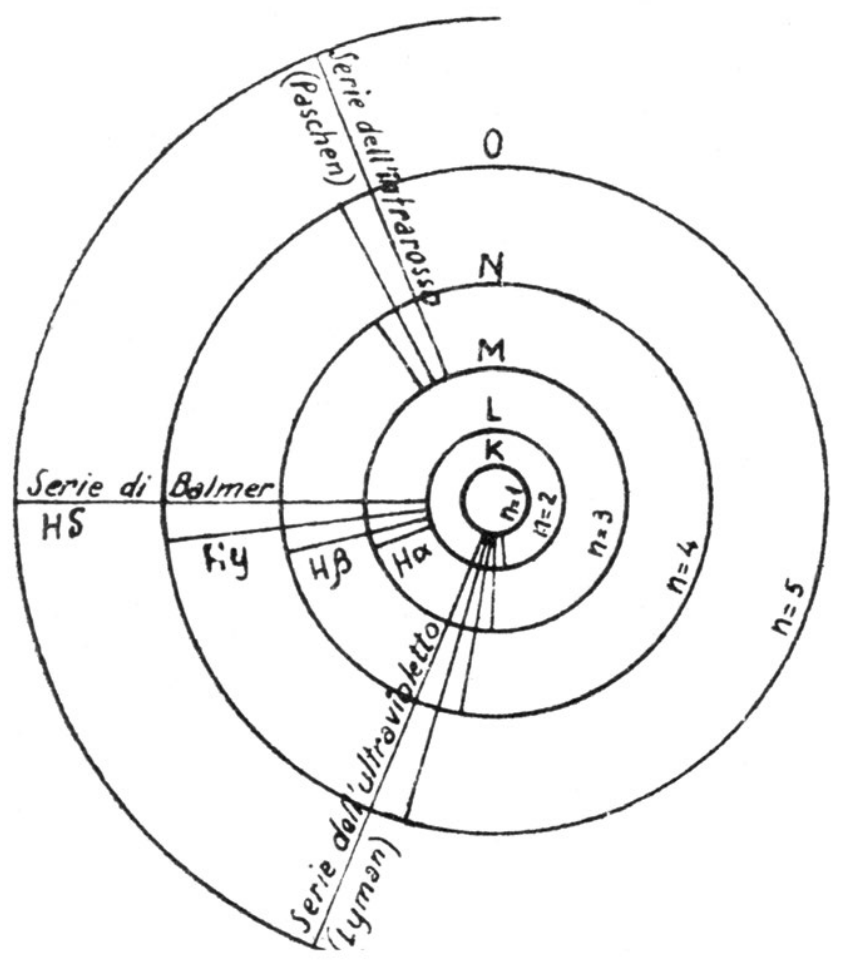

Fig. 7. Le orbite di Bohr (per l'atomo di idrogeno non in scala) e transizioni corrispondenti alle varie serie.

Per la conservazione dell'energia doveva allora aversi

$$
E_{n}-E_{n^{\prime}}=h v
$$

e quindi le frequenze emesse (o assorbita) essere determinate dai valori dei livelli energetici $E_{n}$. 
Bohr affrontò specificamente il problema dell'atomo di idrogeno costituito da un nucleo di carica $e_{0}$ circa 2000 volte più pesante e un solo elettrone trattenuto attorno al nucleo da una forza puramente coulombiana corrispondente al potenziale $\mathrm{U}(r)=-\frac{e_{0}^{2}}{r}$. Egli ammise che l'elettrone potesse muoversi solo sul orbite circolari il cui raggio fosse specificato dalla regola di quantizzazione:

$$
m v r=n \frac{h}{2 \pi}(n=1,2, \ldots),
$$

secondo la quale il momento angolare dell'elettrone (prodotto della quantità di moto per la distanza dal nucleo), che ha le stese dimensioni di un'azione, dovesse essere multiplo intero della costante di Planck divisa $2 \pi$ (Fig. 7). Per un dato $n$ un semplice calcolo fornisce per l'energia, sulla base delle leggi della meccanica, il valore

$$
E_{n}=-\frac{2 \pi^{2} e_{0}^{4} m_{e}}{h^{2}} \frac{1}{n^{2}}
$$

Se ora nell'espressione di $E_{n}$ si sostituiscono alla carica, alla massa dell'elettrone e alla costante di Planck i valori noti, applicando la (3) si riproduce quantitativamente l'intero spettro dell'idrogeno con una precisione dell'ordine di 1/10000. Le stesse regole possono essere applicate con lo stesso successo ai cosiddetti atomi idrogenoidi, gli atomi di elementi leggeri contigui all'idrogeno privati di tutti i loro elettroni meno uno, cioè gli ioni $\mathrm{He}^{+}, \mathrm{Li}^{++}, \mathrm{B}^{+++} \ldots$ Questi ultimi differiscono dall'atomo di idrogeno solo per la carica nucleare che in questo caso è uguale a $Z e_{0}$; ciò ha semplicemente l'effetto di moltiplicare l'espressione di $E_{n}$ per $Z^{2}$.

Le regole specifiche proposte da Bohr nel caso dell'atomo di idrogeno, sono naturalmente molto particolari e certamente non immediatamente estendibili ad atomi a più elettroni. Una loro generalizzazione è stata proposta da Ehrenfest (1914) e perfezionata da Sommerfeld (1916). Queste ultime si applicano a un generico sistema a $f$ gradi di libertà completamente integrabile, che ammette $f$ costanti del moto $J_{1}, J_{2}, \ldots J_{f}$ con le dimensioni di un'azione e consiste di nuovo nell'imporre per ciascuna di tali costanti un valore uguale a un multiplo intero di $\frac{h}{2 \pi}$.

Le ipotesi di Sommerfeld sono in particolare soddisfatte nel caso di una singola particella in un potenziale a simmetria sferica $U(\mathrm{r})$, cioè per un potenziale che dipende solo dalla distanza da un centro fisso. Le nuove regole equivalgono ad allargare la classe delle orbite ammissibili 
oltre quelle semplicemente considerate da Bohr. Ogni orbita è in questo caso caratterizzata da tre numeri quantici, $n, l, m$, che possono assumere i seguenti valori

$$
n=1,2,3, \ldots \quad l=0,1, \ldots n-1 \quad m=-l,-l+1, . . l .
$$

Il numero $n$, detto numero quantico principale, può assumere qualsiasi valore positivo intero ed è da identificarsi con quello proposto da Bohr. Per ogni fissato $n$ il numero $l$ può assumere invece solo $n$ valori distinti ed è questo numero che ora specifica il valore del modulo del momento angolare e la forma dell'orbita. Per $n$ e $l$ fissati, infine, $m$ può assume $2 l+1$ valori distinti e rappresenta il valore della componente del momento angolare in unità $\frac{h}{2 \pi}$ lungo una direzione di riferimento (comunemente identificata con l'asse $z$ del sistema cartesiano) e, quindi, la disposizione dell'orbita nello spazio.

$\mathrm{Nel}$ caso di un generico potenziale a simmetria sferica, l'energia che compete a ciascuna orbita dipende solo dai numeri $n$ e $l$. Nel caso di un potenziale coulombiano puro $U(r)=-\frac{Z e^{2}}{r}$ ancora soltanto dal numero $n$ e ha un'espressione identica a quella ottenuta da Bohr. La rottura della degenerazione in $l$ nel caso più generale permette, però, di spiegare la struttura molto più complessa dello spettro dei metalli alcalini.

Questi ultimi, che nella tavola degli elementi seguono immediatamente i gas nobili, sono caratterizzati da un elettrone più esterno debolmente legato ed è a tale elettrone (elettrone ottico) che si attribuisce l'emissione o l'assorbimento di radiazione nella regione attorno al visibile. Esso si suppone soggetto ad un potenziale efficace a simmetria sferica che risulta dalla somma del potenziale coulombiano puro esercitato dal nucleo e da uno repulsivo dovuto all'azione media degli altri elettroni che si ammette realizzino nel loro insieme una distribuzione di carica a simmetria sferica. L'effetto di una tale distribuzione è quello di schermare in misura maggiore o minore l'azione del nucleo a seconda della profondità in cui ci si pone nella nube elettronica. Detto potenziale efficace dovrà quindi interpolare una sua espressione vicino al nucleo, dove l'azione schermante degli altri elettroni è trascurabile e una a grande distanza dove l'azione di schermo è massima. Avremo quindi

$$
U(r) \rightarrow \begin{array}{ll}
-\frac{Z e^{2}}{r} & \text { per } r \rightarrow 0 \\
-\frac{e^{2}}{r} & \text { per } r \rightarrow \infty
\end{array}
$$


Per un potenziale di questa forma i livelli energetici hanno qualitativamente una disposizione del tipo indicato nella Fig. 8 con un'energia che cresce al crescere di $n$ e per un dato $n$ al crescere di $l$. Nella figura i tratti orizzontali rappresentano (non in scala) i valori delle energie dei singoli livelli; questi ultimi sono disposti per chiarezza su colonne diverse e sono contrassegnati con un numero che rappresenta il valore di $n$ e una lettera che corrisponde ai valori di $l$. Precisamente le lettere $s, p, d, f, \ldots$ indicano le orbite corrispondenti rispettivamente ai valori $l$ $=0,1,2,3, \ldots$ Per $n=1$ può essere solo $l=0$, e quindi abbiamo una sola orbita di tipo $s$; per $n=2$, può essere $l=0,1$ e quindi un'orbita di tipo $s$ e tre di tipo $p$; per $n=3$ un'orbita di tipo $s$, tre di tipo $p$, cinque di tipo $d$; per $n=4$ oltre a quelle di tipo $s, p, d$ ne abbiamo ancora sette di tipo $f$ ecc.

Questa maggiore ricchezza di livelli insieme a certe regole di selezione, conseguenza delle leggi di conservazione, che restringono la classe delle transizioni possibili, permettono di ricostruire, almeno qualitativamente, l'intera struttura dello spettro. Ciò è possibile però al prezzo di ammettere, contro ogni aspettativa, che lo stato fondamentale dell'elettrone ottico del litio sia uno stato $2 s$ e non $1 s$; quello del sodio uno stato $3 s$, quello del potassio uno stato $4 s$ ecc., non siano cioè i più bassi previsti. Questo fatto, come vedremo, si rivelerà della più grande importanza.

Va infine ricordato che, se si sottopone il metallo alcalino in studio ad un campo magnetico, anche la degenerazione nel numero quantico $m$ viene rotta, il numero di livelli cresce quindi ulteriormente e ci si dovrebbe attendere che righe spettrali originariamente singole si scindano in più componenti. Il fenomeno, ben noto, (effetto Zeeman) si presenta effettivamente, ma la ricchezza di righe osservata è molto maggiore di quella che si può prevedere sulla base dei valori di $m$ ammissibili. Per dar ragione di questo fatto, nel 1925 Goudsmit e Uhlembeck proposero di attribuire all'elettrone anche uno spin, un momento angolare intrinseco (un moto di rotazione di questo su se stesso in un modello di elettrone esteso), che come il momento angolare dovuto al moto orbitale supponevano quantizzato. Precisamente ammettevano che la proiezione della componete di questo sulla direzione di riferimento potesse assumere nelle consuete unità $\frac{h}{2 \pi}$ due soli valori, $m_{S}= \pm \frac{1}{2}$. 


\section{Energia di ionizzazione}

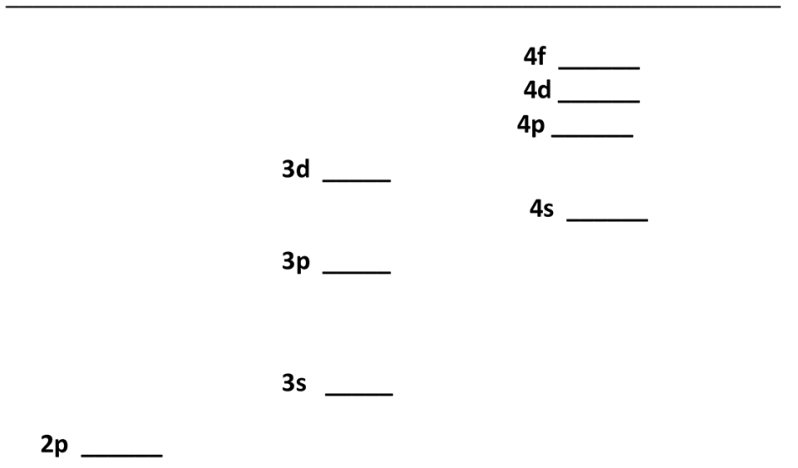

$\uparrow$ Energia

$2 s$

1s

Fig. 8. Disposizione schematica (non in scala) dei livelli energetic per un potenziale del tipo dell'eq. (6).

Per facilitare il confronto con la Meccanica Quantistica che è stata successivamente sviluppata, d'ora in avanti alla disposizione dell'elettrone su una certa orbita con una data orientazione dello spin ci riferiremo come allo stato dell'elettrone. Questo sarà quindi caratterizzato dai quattro numeri quantici $n, l, m, m_{s}$ e l'aggiunta del quarto numero porterà a un raddoppio di tutti gli stati individuati dai primi tre.

Sempre nel 1925 W. Pauli, con riferimento proprio all'assenza inattesa degli stati prevedibili di energia più bassa dell'elettrone ottico nei metalli alcalini, enunciava il suo principio di esclusione, secondo il quale due elettroni non possono mai trovarsi sul medesimo stato o, equivalentemente, possedere numeri quantici identici e formulava un modello di atomo ad elettroni indipendenti che permette una prima sostanziale comprensione del significato del sistema periodico.

A questo proposito va sottolineato che neppure le regole di Sommerfeld sono applicabili al caso di un atomo a più elettroni trattato come sistema unitario. Riprendendo idee già abbozzate da Bohr, tuttavia, Pauli propose un modello di atomo a elettroni indipendenti, 
in cui, non solo un ipotetico elettrone più esterno, ma ogni elettrone fosse supposto sottoposto ad un potenziale medio del tipo (6). Secondo tale modello lo stato complessivo dell'atomo è individuato dalla disposizione degli elettroni sui vari stati possibili nel rispetto del suddetto principio di esclusione.

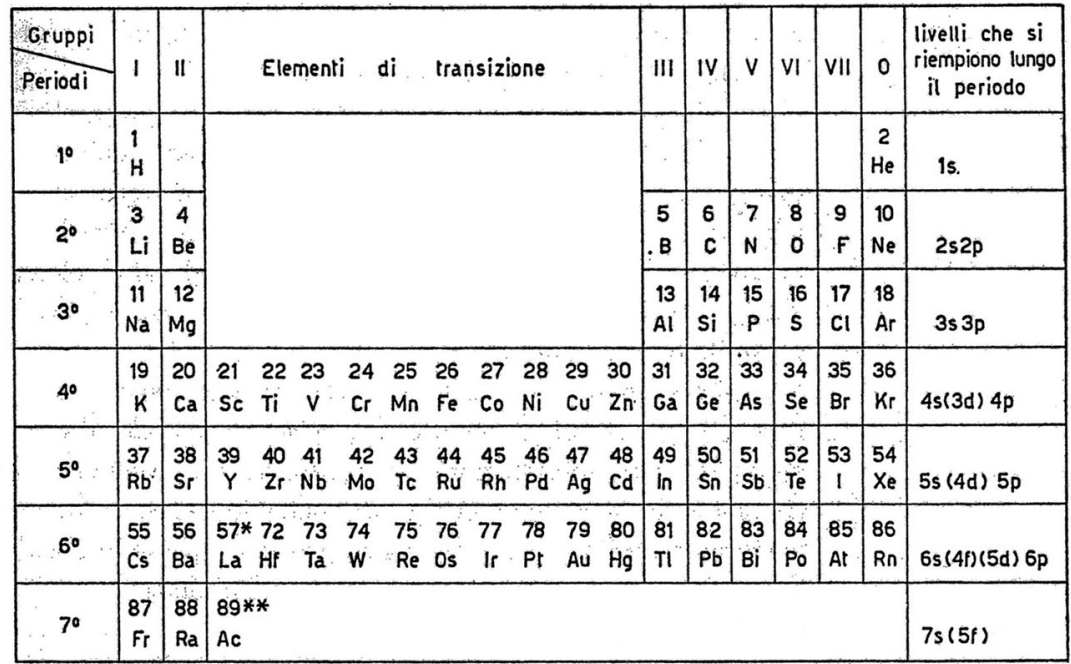

\begin{tabular}{|c|c|c|c|c|c|c|c|c|c|c|c|c|c|c|}
\hline * Lantanidi & 58 & 59 & 60 & 61 & 62 & 63 & 64 & 65 & 66 & 67 & 68 & 69 & 70 & 71 \\
$4 f$ & $\mathrm{Ce}$ & $\mathrm{Pr}$ & $\mathrm{Nd}$ & $\mathrm{Pm}$ & $\mathrm{Sa}$ & $\mathrm{Eu}$ & $\mathrm{Gd}$ & $\mathrm{Tb}$ & $\mathrm{Dy}$ & $\mathrm{Ho}$ & $\mathrm{Er}$ & $\mathrm{Tm}$ & $\mathrm{Yb}$ & $\mathrm{LU}$ \\
\hline
\end{tabular}

\begin{tabular}{|c|c|c|c|c|c|c|c|c|c|c|c|c|c|c|}
\hline$* *$ Attinidi & 90 & 91 & 92 & 93 & 94 & 95 & 96 & 97 & 98 & 99 & 100 & 101 & 102 & 103 \\
\hline $5 f$ & $\mathrm{Th}$ & $\mathrm{Pa}$ & $\mathrm{U}$ & $\mathrm{Np}$ & $\mathrm{Pu}$ & $\mathrm{Am}$ & $\mathrm{Cm}$ & $\mathrm{Bk}$ & $\mathrm{Cl}$ & $\mathrm{Es}$ & $\mathrm{Fm}$ & $\mathrm{Md}$ & $\mathrm{No}$ & $\mathrm{Lw}$ \\
\hline
\end{tabular}

Fig. 9. Tavola periodica di Bohr, detta a periodi lunghi.

In particolare, nello stato fondamentale gli elettroni devono sistemarsi negli stati disponibili di energia più bassa, tenendo presente che su uno stato di tipo $s$ possono trovare posto al massimo due elettroni in corrispondenza ai due diversi valori di $m_{s}$, su uno di tipo $p$ sei elettroni, 10 su uno di tipo $d, 14$ su uno di tipo $f$, ecc.

La disposizione progressiva degli elettroni negli atomi dei vari elementi, che risulta dai precedenti criteri è messa in evidenza nel modo migliore dalla tavola a lunghi periodi, come proposta da Bohr (Fig. 9) e fornisce un'immediata interpretazione di quest'ultima, solo che si ammetta che le proprietà chimiche degli stessi in condizioni normali siano determinate dalla disposizione degli elettroni più esterni. Si noti 
che in tale tavola elementi con proprietà simili sono disposti sulla stessa colonna e costituiscono quello che è detto un gruppo. Gli elementi disposti sulla stessa riga sono invece detti costituire un periodo perché in essi si ripetono nello stesso ordine elementi con proprietà simili a quelli riportati nella riga sovrastante.

Veniamo ora esplicitamente alla disposizione degli elettroni nei vari elementi come cresce il numero di questi ultimi e cominciamo con l'atomo di idrogeno, $H$. Questo possiede un solo elettrone, che quindi deve disporsi sul livello più basso che secondo la Fig. 8, è di tipo 1 s. Segue l'atomo di elio, $\mathrm{He}$, con due elettroni; anche il secondo si disporrà sul medesimo tipo di livello che come sappiamo può ospitarne due. Con l'elio si chiude il $1^{\circ}$ brevissimo periodo. Passando al $2^{\circ}$ periodo, al Litio, $L i$, e al berillio, $B e$, rispettivamente con tre e quattro elettroni, i primi due si disporranno ancora nello stato $1 s$; quelli successivi, essendo ormai l' $1 s$ totalmente occupato, dovranno porsi sul livello $2 s$. Seguono sei elementi, il boro, $B$, il carbonio $C$, ecc. fino al neon, $N e$; in questi gli elettroni oltre i primi quattro devono disporsi sugli stati $2 p$. Nell'ultimo, il neon, un gas nobile, anche tutti gli stati $2 p$ sono occupati, come indicato nell'ultima colonna. $\mathrm{Nel} 3^{\circ}$ periodo, dal sodio $\mathrm{Na}$ all'argon $\mathrm{Ar}$, si riempiono progressivamente prima gli stati, $3 s$ e poi i $3 p$.

Successivamente ci si attenderebbe che comincino a riempirsi gli stati $3 d$. Questi hanno però un'energia minore degli stati $4 p$ ma leggermente superiore a quella degli stati $3 s$. Di conseguenza prima cominciano a riempirsi questi ultimi, e così inizia il $4^{\circ}$ periodo; abbiamo il potassio e il calcio. seguono dieci elementi che corrispondono invece al progressivo riempirsi degli stati $3 d$, sono i cosiddetti metalli di transizione, e poi ancora di nuovo sei elementi dal gallio al cripton che si incolonnano con quelli dei gruppi dal III allo 0 e corrispondono al riempirsi degli stati $4 p$. Una situazione simile si ripete con il $5^{\circ}$ periodo, si riempiono prima gli stati $5 s$, rubidio e stronzio, poi gli stati $4 d$, e si ha quindi una successiva serie di metalli di transizione, e poi gli stati $5 p$. Nel $6^{\circ}$ periodo dopo gli stati $6 s$, col lantanio, $L a$, cominciano a riempirsi gli stati $5 d$, ma subito dopo segue una serie di quattordici elementi, detti lantanidi o terre rare, che corrispondono al riempirsi degli stati $4 f$, finiscono di riempirsi quindi gli stati $5 d$ e poi i $6 p$. Di nuovo la situazione si ripete con l'ultimo periodo, con l'inserimento dei cosiddetti attinidi ed elementi tutti radioattivi e in gran parte artificiali, che non esistono in natura. Esistono in realtà alcune eccezioni alla disposizio- 
ne ideale che abbiamo descritto, dovute ai limiti stessi del modello, ad esempio il rame, l'argento e l'oro hanno un solo elettrone sullo stato $s$ più esterno mentre i sottostanti stati $d$ sono tutti occupati. Questo assimila per alcune proprietà questi elementi a quelli del I gruppo.

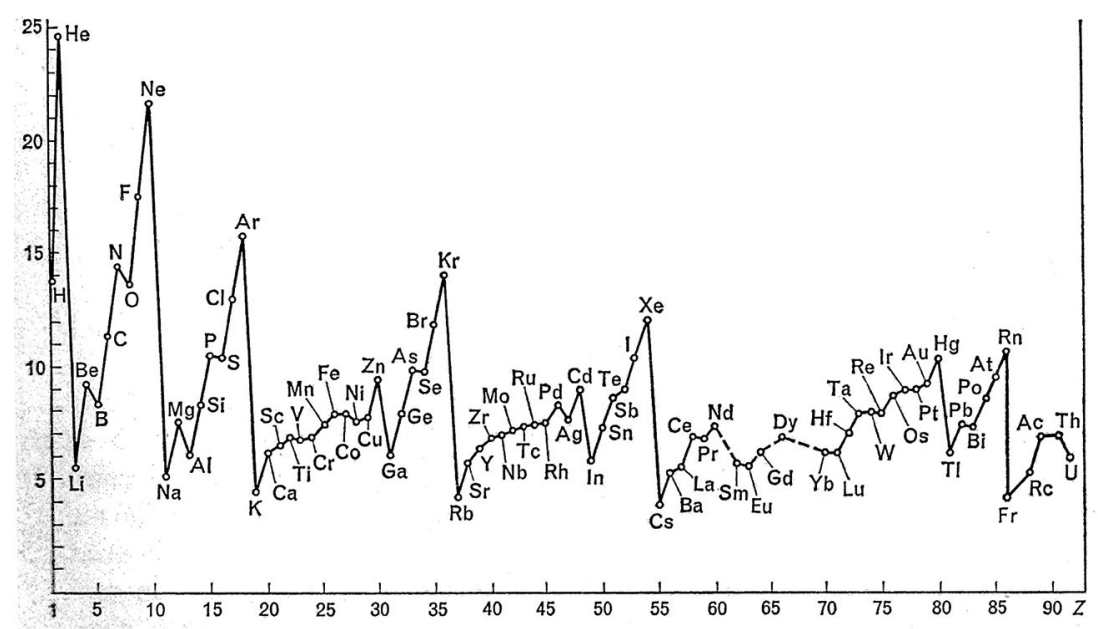

Fig. 10. Potenziale di prima ionizzazione in funzione di $Z$.

Si noti che con l'aumentare di $Z$, e quindi della carica nucleare i livelli riportati in Fig. 8, si approfondiscono complessivamente in maniera continua mantenendo però approssimativamente la stessa disposizione reciproca, le proprietà di un elemento all'interno del medesimo gruppo variano perciò gradualmente. Nel passaggio invece da un elemento al successivo nell'ambito dello stesso periodo, al crescere del numero degli elettroni su un certo tipo di stato e soprattutto al riempirsi di tutti gli stati di una certa classe, si hanno discontinuità anche drastiche. La situazione è molto bene illustrata dal grafico della Fig. 10 in cui sono riportati i potenziali di prima ionizzazione, cioè le energie minime, espresse in $\mathrm{eV}$ $(\text { elettroni-volt })^{1}$ necessarie per strappare dall'atomo un primo elettrone,

1 L'ellettron-volt (eV) rappresenta l'energia acquistata da un elettrone quando viene accelerato tra due placche tra cui esiste una differenza di potenziale di un Volt, $1 \mathrm{eV}=1,6 \cdot 10^{-19} \mathrm{~J}$. 
equivalente quindi all'energia cambiata di segno dell'elettrone che si trova sul livello più esterno. Si noti che la curva presenta dei massimi molto marcati in corrispondenza dei gas nobili, gli elementi più stabili. Si notino anche: la crescita dall'idrogeno all'elio dovuta all'approfondirsi del livello $1 s$ al crescere della carica nucleare, il

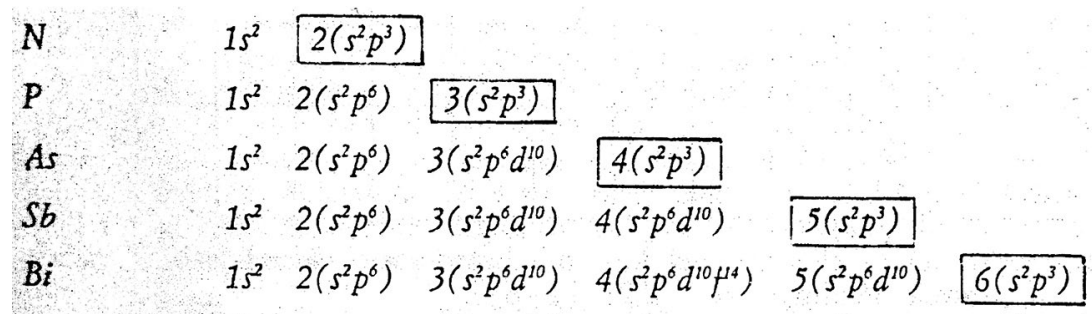

Fig. 11. Disposizione degli elettroni negli elementi del V gruppo. Gli esponenti attribuiti al simbolo degli stati stanno ad indicare il numero di elettroni disposti su quello stato. Si noti che la configurazione elettronica pù esterna è sempre del tipo $s^{2} p^{3}$, cioè due elettroni nel più esterno stato di tipo $s$ e tre nello stato di tipo $p$. (da P. Silvestroni, Fondamenti di Chimica, $6^{\circ}$ ed. ; Veschi, Roma 1980.

minimo in corrispondenza del litio quando comincia a riempirsi lo stato $2 s$, la risalita dal litio al berillio, il nuovo minimo in corrispondenza del boro, quando comincia a riempirsi lo stato $2 p$ e la risalita fino al neon. La situazione si ripete nei periodi successivi. E' notevole in particolare l'andamento poco strutturato in corrispondenza dei metalli di transizione. Minori irregolarità sono dovute alle interazioni che coinvolgono lo spin.

Abbiamo sopra supposto che le proprietà chimiche di un elemento in condizioni ordinarie siano determinate dalla disposizione degli elettroni sugli stati più esterni, in particolare sugli stati $s$ e $p$. Osserviamo che gli elementi appartenenti allo stesso gruppo, cioè disposti sulla stessa colonna, sulla base del modello precedente, si trovano effettivamente in questa condizione. In Fig. 11, a titolo d'esempio, è riportata la distribuzione completa degli elettroni per gli elementi del gruppo V dell'azoto.

L'irraggiamento X al contrario dipende certamente solo dagli elettroni più interni, i cui livelli sono molto maggiormente spaziati. Essi sono praticamente soggetti all'intera carica nucleare, i loro livelli hanno perciò la stessa espressione di quella degli atomi idrogenoidi, cioè, come abbiamo detto, la (4) moltiplicata per, $Z^{2}$. La stessa dipendenza dovranno quindi avere le frequenze della radiazione emessa e resta così giustificata la stessa legge di Moseley. 


\section{La MeCCANica Quantistica}

Come abbiamo visto, con il modello di Pauli nel contesto della "vecchia" Teoria dei Quanti si ha una prima sostanziale comprensione del significato della Tavola degli elementi. La "vecchia" Teoria dei Quanti era però una teoria sostanzialmente incongrua, perché da una parte utilizzava ancora la meccanica e l'elettromagnetismo classico, dall'altra li contraddiceva introducendo delle regole di quantizzazione ad hoc. Particolarmente ad hoc risultava il principio di esclusione. Nel contesto della vecchia teoria, inoltre, non era possibile alcuna comprensione della natura del legame molecolare e l'idea che le proprietà chimiche fossero determinate dalla corteccia elettronica più esterna restava completamente ingiustificata. Queste difficoltà sono completamente superate con la nuova Meccanica Quantistica sviluppata dal 1924 al 1927 e poi con la successiva Teoria Quantistica dei Campi che ne costituisce l'indispensabile sviluppo relativistico.

Nella sua forma più intuitiva, la cosiddetta Meccanica Ondulatoria, la nuova teoria si è sviluppata da un'intuizione del fisco teorico francese L. De Broglie. Partendo dalle ipotesi di Planck e di Einstein che attribuivano una doppia natura ondulatoria e corpuscolare alla radiazione elettromagnetica, De Broglie propose che, reciprocamente, alle particelle cosiddette materiali dovesse essere associata un'onda la cui frequenza $v$ e lunghezza d'onda $\lambda$ fossero legate all'energia e alla quantità di moto della particella dalle relazioni

$$
w=h v, \quad p=\frac{\mathrm{h}}{\lambda},
$$

analoghe alle (1) e alle (2). Si noti che la seconda delle (7) segue in questa forma dalla prima, se si vuole che questa risulti relativisticamente invariante, e che d'altra parte nel caso della luce, dove $\lambda=\frac{c}{v}$, la stessa (2) può essere riscritta in questa forma. In questo contesto i livelli energetici discreti esistenti nell'atomo sono interpretati come conseguenza della proprietà tipica dei fenomeni ondulatori confinati in regioni limitate dello spazio di ammettere solo delle frequenze discrete $v_{1}, v_{2}$, $\ldots v_{n}, \ldots$, , v. Fig. 12) da cui, per la relazione di Planck

$$
W_{n}=h v_{n} .
$$


De Broglie non riuscì ad andare al di là di considerazioni qualitative. Le sue idee furono però riprese da Schroedinger, che riuscì a proporre un'equazione non relativistica che ne realizza le ipotesi

$$
\left[-\frac{\hbar}{2 m} \nabla^{2}+U(x)\right] \psi(x, t)=i \hbar \frac{\partial}{\partial t} \psi(x, t) .
$$

In questa $U(\boldsymbol{x})$ rappresenta sempre il potenziale che agisce sulla particella, $\psi(x, t)$ la grandezza vibrante (espressa da un numero complesso) nella sua dipendenza dalla posizione e dal tempo e $\boldsymbol{x} \equiv(x, y, z)$ sta per l'insieme delle coordinate che specificano la posizione.

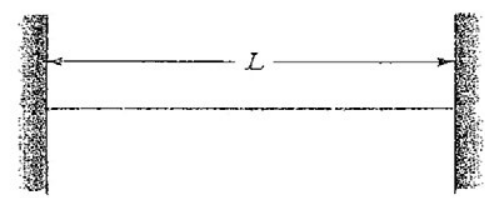

(a)

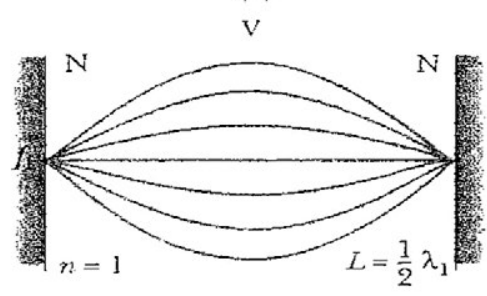

(b)

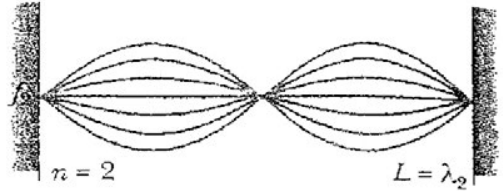

(c)

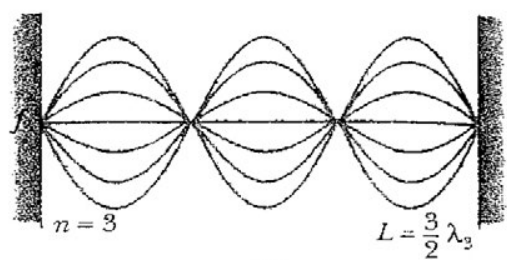

(d)

Fig. 12. Modi fondamentali di vibrazione di una corda tesa tra due estremi fissi, tipicamente una corda di violino (a). Tutte le vibrazioni della corda sono esprimibili come sovrapposizione di onde monocromatiche stazionarie (che singolarmente non si propagano) che hanno certe frequenze specifiche. Intuitivamente un' onda che si propaghi sulla corda si riflette successivamente sui due estremi e dovrà cancellarsi se dopo ciascuna riflessione non si trova in concordanza di fase con quelle prodotte in riflessioni precedenti. Ciò evidentemente può accadere solo se la lunghezza dell'onda è un sottomultiplo intero del doppio della lunghezza della corda. Abbiamo così una frequenza fondamentale $v_{1}=V / \lambda_{1}$ (V velocità di propagazione di una deformazione sulla corda indefinitamente estesa) rappresentata in (b), e le armoniche $v_{2}=2 v_{1}, \quad v_{3}=3 v_{1}$, ecc. rappresentate in (c), (d), ... Un fenomeno del tutto simile si presenta anche in tre dimensioni per un qualunque fenomeno ondulatorio confinato in una regione limitata dello spazio, come ad esempio il suono all'interno di una cassa armonica o la radiazione elettromagnetica all'interno di una cavità metallica. 
Schroedinger fu anche capace di risolvere la (9) per un potenziale coulombiano puro $U=-\frac{e^{2}}{r}$, quindi nel caso dell'atomo di idrogeno, e determinarne le frequenze caratteristiche ritrovando per i livelli energetici un'espressione identica alla (4), data da Bohr. ${ }^{2}$ Per un generico potenziale a simmetria sferica le soluzioni stazionarie sono poi specificate da una terna di numeri quantici $n, l, m$ del tutto analoghi a quelli introdotti nella vecchia teoria (una tale struttura è in effetti determinata in entrambi i casi dalla simmetria sferica del problema), che ora naturalmente specificano delle funzioni d'onda. Le corrispondenti frequenze caratteristiche e quindi i livelli energetici dipendono ancora solo dai numeri $n$ e $l$. Per un potenziale del tipo (6) inoltre questi livelli possono essere calcolati in modo approssimato e si trova un risultato qualitativamente del tutto simile a quello ottenuto con le regole di Sommerfeld e rappresentato nella Fig. 8.

Finalmente per poter tener conto nel contesto dell'equazione (9) della possibilità di uno spin e di termini che esprimessero interazioni dipendenti da tale grandezza, Pauli introdusse nell'espressione della funzione d'onda una ulteriore coordinata $\omega$ con due possibili valori riscrivendo quind la funzione d'onda come $\psi(\boldsymbol{x}, \omega, t)$ in luogo semplicemente di $\psi(\boldsymbol{x}, t)$.

Veniamo ora al problema di una conferma della natura ondulatoria delle particelle. Come nel caso della luce, questa natura e la correttezza dell'equazione (7), possono essere verificate con esperimenti di interferenza. Nel caso della luce un classico esperimento di interferenza è quello di Young del 1802, ottenuto facendo incidere un fascio di luce su un diaframma A (Fig. 13) su cui sono praticate due fenditure molto ravvicinate e raccogliendo la figura che ne risulta su un secondo schermo B. Quest'ultima consiste nell'alternanza di fasce scure e luminose parallele alle fenditure. Poiché la lunghezza dell'onda associata ad un elettrone con

2 Più precisamente per un potenziale che si annulli all'infinito la (9) possiede due tipi di soluzioni. Un primo tipo, che corrisponde ad un'energia positiva della particella, consiste in pacchetti d'onda sovrapposizione di onde monocromatiche di frequenza qualsiasi, che si avvicinano ma poi si allontanano indefinitamente dalla regione in cui il potenziale è importante. Un secondo tipo è costituito da onde monocromatiche singole, che si annullano rapidamente fuori dal raggio d'azione del potenziale e corrispondono ad un'energia negativa della particella e quindi ad un suo stato legato. E' per queste seconde che si presenta il fenomeno delle frequenze discrete.

3 L'elettronvolt $(\mathrm{eV})$ è l'unità di energia che con i suoi multipli si usa nella 
un'energia di $100 \mathrm{eV}^{3}$ è di $1,2 \cdot 10^{-8} \mathrm{~cm}$, cioè solo dell'ordine delle dimensioni atomiche i primi esperimenti di verifica furono di diffrazione su cristalli. Per esperimenti del tipo di Young con un fasci di elettroni si è dovuto attendere fino alla seconda metà del ' 900 , questi hanno tuttavia concettualmente particolare interesse.

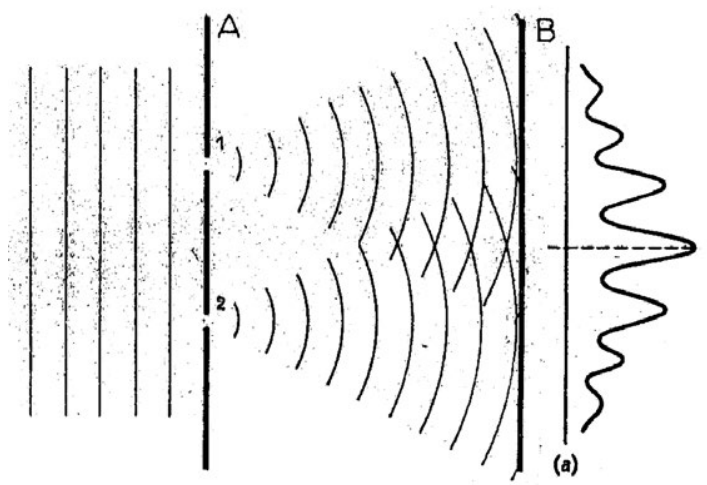

Fig. 13. Esperimento del tipo di Young. Un fascio di luce o di particelle investe il diaframma A su cui sono praticate due fenditure parallele; sullo schermo B si forma una figura di interferenza costituita da un alternarsi di fasce scure e luminose che corrisponde al sommarsi o sottrarsi delle ampiezze delle due onde provenienti dalle due fenditure a seconda che esse, in dipendenza dal loro diverso cammino, si trovino nel punto considerato in concordanza o opposizione di fase. La figura, schematicamente rappresentata in (a), dalla curva di intensità può essere registrata su una lastra fotografica che è un rivelatore integrale, nel senso che non distingue i singoli fotoni o particelle ma ne rileva solo il flusso complessivo, o anche ricostruita a partire dalla distribuzione statistica delle particelle sullo schermo (v. Fig. 14).

Sia per la luce che per particelle materiali si deve comunque parlare, come abbiamo detto, di una doppia natura ondulatoria e corpuscolare. Un fotone o una particella si comportano come un'onda, e quindi oggetti estesi, quando si propagano ma, essendo indivisibili, si presentano come corpuscoli localizzati quando si cerca di rivelarli. Nell'attraversare una rete di contatori in grado di registrarli singolarmente, essi agiscono sempre su uno solo di questi.

Fisica Atomica, nella Fisica Nucleare e in quella delle particelle. $1 \mathrm{eV}=1,6 \quad 10^{-19} \mathrm{~J}$ è l'energia acquistata da un elettrone se accelerato in una differenza di potenziale di $1 \mathrm{~V}$. 

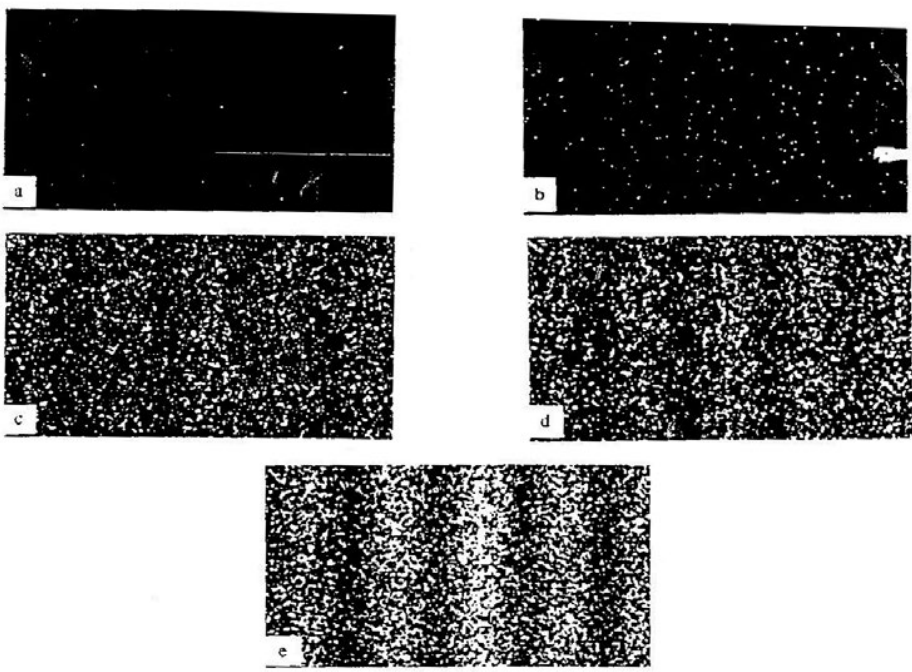

Fig. 14. Esperimento di Tonomura (1989). La figura di interferenza prodotta da un fascio di elettroni che incide su un biprisma (in pratica un filo carico positivamente), ingrandita con ottica elettronica, è raccolta su uno schermo fluorescente. Un reticolo di fotomoltiplicatori disposto davanti allo schermo permette di registrare attraverso strumentazione elettronica le scintillazioni prodotte dalle singole particelle. Si può così osservare come la figura venga progressivamente costruita dal successivo accumularsi delle particelle sullo schermo. Numero degli elettroni corrispondenti alle figure riportate nei vari riquadri: a) 10 elettroni, b) 100 , c) 3000 , d) 20.000 , e) 70.000 (da A.Tonomura et al, American Jornal of Physics, 57, 117, 1989).

Per conciliare questi due aspetti già Einstein nel caso della luce aveva genericamente ammesso che l'intensità di radiazione elettromagnetica macroscopica corrispondesse alla distribuzione statistica nello spazio dei fotoni che la componevano. Questa ipotesi nel caso dell'equazione di Schroedinger è stata ripresa da Born che ne ha fatto un postulato esplicito. Secondo tale postulato l'espressione $|\psi(\boldsymbol{x}, \omega, t)|^{2} d V$ deve essere interpretata come probabilità di trovare la particella entro il volumetto $d V$ attorno al punto $\boldsymbol{x}$ con spin $\omega .{ }^{4}$ In particolare una figura

4 In questa prospettiva la costante moltiplicativa a meno della quale la $\psi$ è sempre determinata (essendo l'equazione (9) lineare) va scelta in modo che la probabilità di trovare la particella in qualunque punto dello spazio con qualunque valore dello spin sia uguale ad 1. 
di interferenza, del tipo di Youmg (Fig. 13) o altro, dovrebbe sempre risultare dalla distribuzione statistica delle particelle sullo schermo. Con i progressi della miniaturizzazione e dell'elettronica moderna questa circostanza ha potuto essere verificata in maniera diretta per i fotoni, per gli elettroni (Fig. 14) e, più di recente, anche per particelle più pesanti.

L'equazione (9) si riferisce ad una sola particella in un campo di forze esterno. Schroedinger è stato anche capace di estenderla a un sistema di $N$ particelle. A questo scopo ha dovuto però associare al sistema stesso una funzione d'onda definita sull'intero spazio delle configurazioni, cioè una funzione del tipo

$$
\psi\left(\boldsymbol{x}_{1}, \omega_{1} ; \boldsymbol{x}_{2}, \omega_{2} ; \ldots \boldsymbol{x}_{\mathrm{N}}, \omega_{\mathrm{N}} ; t\right),
$$

dipendente dalle posizioni e, naturalmente, nella generalizzazione di Pauli, dalle coordinate di spin di ciascuna singola particella. L'espressione

$$
\left|\psi\left(\boldsymbol{x}_{1}, \omega_{1} ; \boldsymbol{x}_{2}, \omega_{2} ; \ldots \boldsymbol{x}_{\mathrm{N}}, \omega_{\mathrm{N}} ; t\right)\right|^{2} d V_{1} d V_{2} \ldots d V_{N}
$$

è allora interpretata come probabilità di osservare la prima particella entro il volume $d V_{1}$ e con orientazione dello spin $\omega_{1}$, la seconda entro il volume $d V_{2}$ con orientazione dello spin $\omega_{2}$ ecc. In questa equazione più generale, oltre a un eventuale potenziale esterno, quello esercitato dal nucleo nel caso degli elettroni di un atomo, compaiono i potenziali di interazione tra le particelle del sistema.

Osserviamo tuttavia che, nello spirito del formalismo, ad un sistema di particelle identiche deve corrispondere una distribuzione della probabilità complessiva (11) che non distingue tra le stesse ed è quindi simmetrica rispetto ad una qualsiasi permutazione di queste. Questa circostanza si può realizzare sostanzialmente in due modi, supponendo che la funzione d'onda (10) sia essa stessa completamente simmetrica rispetto a una tale permutazione o anche che sia completamente antisimmetrica rispetto alla stessa, cioè che cambi segno in corrispondenza a ogni singolo scambio tra due particelle. E' dunque a questi due tipi di funzioni d'onda che ci dobbiamo in questo caso restringere. Poiché poi l'insieme di tutte le particelle di un determinato tipo esistenti nell'universo dovrebbe in linea di principio essere descritto da un'unica funzione d'onda, il carattere di simmetria o antisimmetria della stessa deve 
essere una proprietà specifica del tipo di particelle. Come si può comprendere, questo carattere ha delle conseguenze molto importanti sulle proprietà collettive dei sistemi. Le particelle possono perciò essere classificate in due grandi categorie, quella dei bosoni (che soddisfano, come anche si dice, la statistica di Bose-Einstein) con funzione d'onda completamente simmetrica e quelle dei fermioni (che soddisfano la statistica di Fermi-Dirac) con funzione d'onda completamente antisimmetrica.

Passiamo ora al problema dell'atomo a $Z$ elettroni nel contesto discusso. A questo scopo noi dovremmo cercare soluzioni dell'equazione per gli elettroni in presenza del potenziale attrattivo del nucleo e di quello di repulsione tra gli stessi elettroni, che abbiano la simmetria ad essi appropriata. Un tale problema non è però risolvibile esattamente ed è necessario perciò procedere con metodi di approssimazione successiva. L'approssimazione di base è analoga a quella adottata nel contesto della vecchia teoria. Si considera un modello a elettroni indipendenti in cui ogni elettrone è soggetto ad un potenziale del tipo dell'equazione (6). Ad ogni elettrone viene in tal modo associata una propria funzione d'onda $\psi_{n l m m_{S}}(\boldsymbol{x}, \omega, t)$, specificata dai numeri quantici $n, l, m, m_{s}$ del tutto corrispondenti, come abbiamo detto, a quelli usati nel modello di BohrSommerfeld, e lo stato complessivo dell'atomo può essere ancora caratterizzato indicando come si distribuiscono gli elettroni tra i vari stati.

Passando alla funzione d'onda complessiva del tipo (10), prescindendo dalle considerazioni di simmetria di cui sopra la più naturale scelta sarebbe semplicemente quella del prodotto delle funzioni d'onda dei singoli elettroni

$$
\psi_{a_{1} a_{2} \ldots a_{\mathrm{Z}}}(1,2, \ldots Z)=\psi_{a_{1}}(1) \psi_{a_{2}}(2) \ldots \psi_{a_{Z}}(\mathrm{Z}),
$$

dove per brevità i numeri $1,2, \ldots$ indicano l'insieme delle coordinate di posizione e di spin della prima particella, $\boldsymbol{x}_{1}, \omega_{1}$, della seconda. $\boldsymbol{x}_{2}, \omega_{2}$ ecc. e gli indici $a_{1} a_{2} \ldots$ stanno per i rispettivi insiemi di numeri quantici, $n_{1}, l_{1}, m_{1}, m_{s 1} ; n_{2}, l_{2}, m_{2}, m_{s 2} ; \ldots . \mathrm{La}(12)$ non rientra però in generale in nessuna delle due classi di simmetria che abbiamo detto possibili. Osserviamo tuttavia che per l'identità degli elettroni tutte le funzioni d'onda ottenute dalla (12) con una perturbazione dell'ordine in cui essi sono presi, corrisponde alla stessa energia totale che è espressa dalla somma delle energie dei singoli stati. Possiamo allora ottenere una funzione d'onda completamente simmetrica nell'ambito dell'approssima- 
zione considerata semplicemente sostituendo alla (12) con la somma di tutte le espressioni dello stesso tipo estesa alle possibili permutazioni (con una ridefinizione naturalmente del fattore complessivo). Una funzione completamente antisimmetrica si potrà invece ottenere con l'analoga somma tipo attribuendo però a ciascun termine un segno + , se la permutazione a cui si riferisce è di tipo pari (cioè se si può ottenere con un numero pari di scambi di coppie), un segno - , se è di tipo dispari.

Con riferimento in particolare al secondo caso, osserviamo che una funzione del tipo descritto si può porre molto semplicemente sotto forma di determinante

$$
\psi_{a_{1} a_{2} \ldots a_{Z}}(1,2, \ldots Z)=\frac{1}{\sqrt{Z !}}\left|\begin{array}{cccc}
\psi_{a_{1}}(1) & \psi_{a_{2}}(1) & \ldots & \psi_{a_{Z}}(1) \\
\psi_{a_{1}(2)} & \psi_{a_{2}}(2) & \ldots & \psi_{a_{Z}}(2) \\
\ldots & \ldots & \ldots & \ldots \\
\psi_{a_{1}}(Z) & \psi_{a_{2}}(Z) & \ldots & \psi_{a_{Z}}(Z)
\end{array}\right|
$$

Notiamo allora in particolare che, se nella (13) due sistemi di numeri quantici sono identici, per esempio $a_{1}=a_{2}$ (ciò che significa $n_{1}=n_{2}, l_{1}$ $=l_{2}, m_{1}=m_{2}, m_{s 1}=m_{s 2}$ ), il determinante ha due colonne uguali e quindi è identicamente nullo. Ciò significa che non esiste in questo caso una funzione d'onda completamente antisimmetrica corrispondente a quel valore dell'energia. I fermioni quindi soddisfano un principio di esclusione e il postulato di Pauli che gli elettroni in un atomo soddisfino un tale principio si può sostituire con quello del tutto coerente nel formalismo della meccanica quantistica che gli elettroni siano fermioni.

Tutte le acquisizioni e le considerazioni qualitative della sezione precedente restano perciò perfettamente valide. Va detto inoltre che i metodi di approssimazione successiva cui sopra abbiamo accennato permettono ora una valutazione anche quantitativa e, quando praticabili, molto precisa del valore dei livelli energetici quale risulta dalla spettroscopia.

In aggiunta la Meccanica quantistica fornisce una teoria del legame molecolare. Questo è visto come conseguenza dell'alterazione che subiscono le funzioni d'onda degli elettroni più esterni e i livelli energetici corrispondenti quando due atomi vengono accostati, così che gli elettroni dell'uno cominciano a sentire l'azione del nucleo e degli elettroni dell'altro. Anche l'ipotesi che le proprietà chimiche di un elemento siano determinate dallo stato degli elettroni più esterni viene così giustificata. 


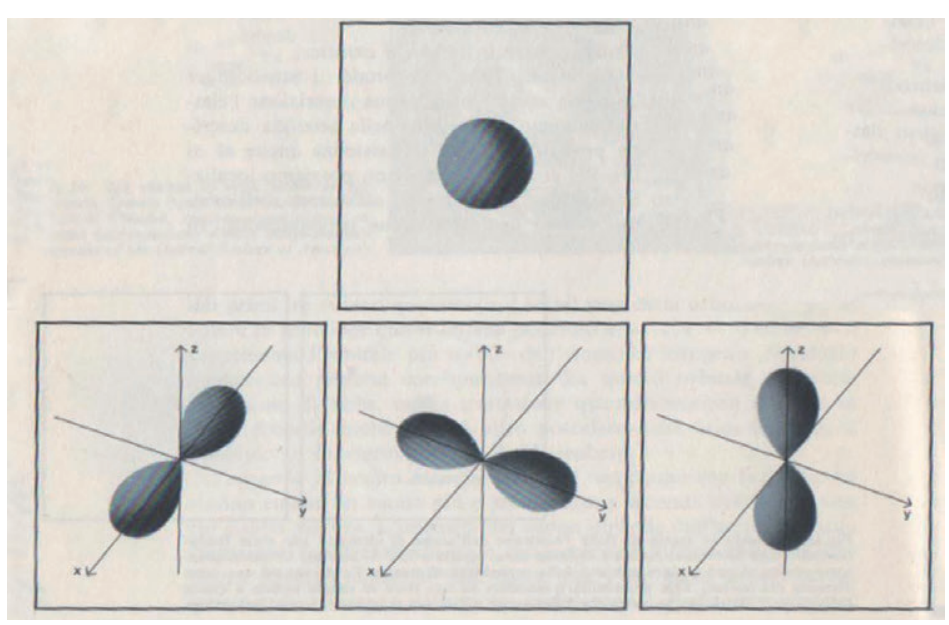

Fig. 15. Rappresentazione schematica della distribuzione di probabilità per le funzioni d'onda $1 s$ e $2 p$ in forma reale. La parte ombreggiata corrisponde alla regione dello spazio delimitata da una superficie di equiprobabilità entro cui la particella deve trovarsi con il $90 \%$ di probabilità (da G. NATTA e M. FARINA, Stereochimica, molecole in 3D, Mondadori, Milano, 1968).

Si deve poi notare a quest'ultimo proposito che la distribuzione di probabilità corrispondente a ciascuna funzione d'onda è a simmetria sferica solo nel caso degli stati $s(l=0)$, ha invece una simmetria assiale nel caso degli stati $p(l=1)$, e ha una struttura ancora più complessa per gli $l$ successivi. Nelle Figg. 15 e 16 sono schematicamente rappresentate le geometrie delle distribuzioni di probabilità relative ad alcune delle funzioni d'onda prese in forma reale corrispondenti ai più bassi valori dei numeri quantici. Si noti la struttura a due lobi allineati su ciascuno dei tre assi coordinati per gli stati $2 p$ e quella a quattro lobi per gli stati $3 d$. Tali strutture determinano le possibili disposizioni spaziali degli atomi nella molecole, le conformazioni di queste ultime. Questa circostanza è di particolare importanza per comprendere le proprietà delle molecole organiche e permette, sfruttando la potenza dei moderni calcolatori, di progettare addirittura alcune molecole su base puramente teorica.

5 Delle $2 l+1$ funzioni d'onda indipendenti che corrispondono a dati $n$ e $l$, quando caratterizzate dal numero sopra introdotto, una sola è reale. Le altre sono però a due a due complesse coniugate è perciò possibile sostituirle con loro combinazioni reali. E' in tale forma che esse risultano più utili nello studio del legame molecolare. 

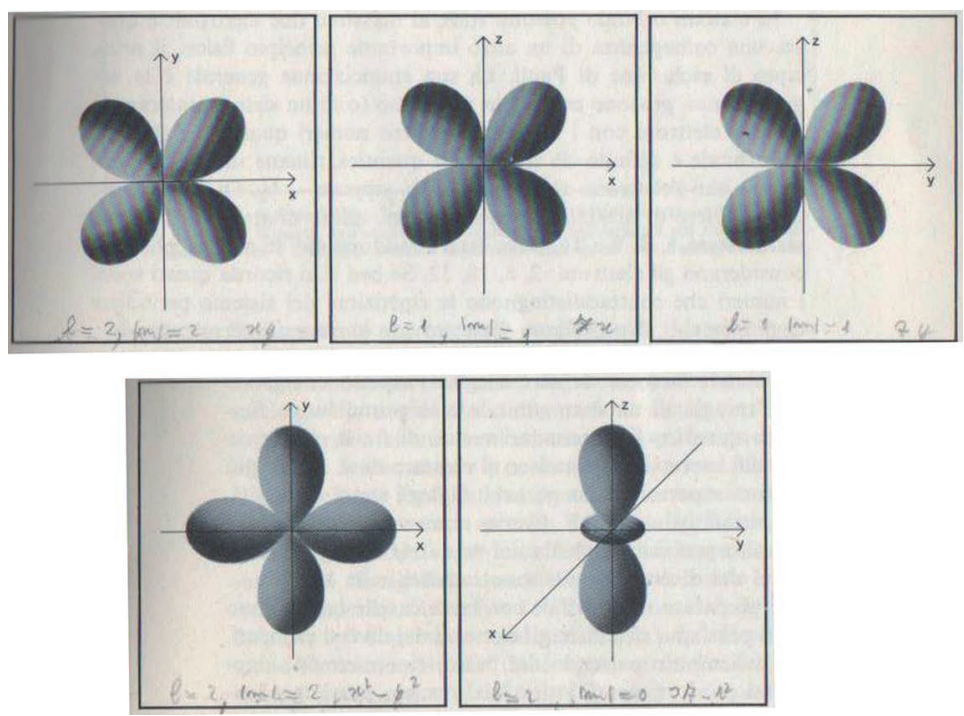

Fig. 16. Rappresentazione schematica delle distribuzioni di probabilità per le funzioni $3 d$ in forma reale (da G. NATTA e M. FARINA, loc. cit.)

\section{La TeOria Quantistica deI CAMPI}

Nella formulazione di Schroedinger della Meccanica Quantistica, a cui ci siamo finora riferiti, le funzioni d'onda hanno evidentemente solo il ruolo di strumenti matematici per previsioni di tipo statistico. Questa circostanza non è in realtà molto in linea con l'originaria intuizione di De Broglie per cui il campo associato alle particelle doveva essere un campo fisico reale come il campo elettromagnetico. Parallelamente alla Meccanica Ondulatoria si era però sviluppata ad opera di W. Heisenberg, M. Born e P. Jordan una seconda formulazione, detta Meccanica delle Matrici, che si riallacciava più direttamente alle regole di Bohr Sommerfeld. Lo stesso Schroedinger aveva poi mostrato la perfetta equivalenza delle due teorie.

Una circostanza importante è che solo quest'ultimo formalismo può essere esteso secondo i requisiti della Relatività, nel cui ambito sono ammesse solo interazioni per contatto. E' solo nel suo contesto che può venire costruita una teoria delle particelle, come il cosiddetto Modello Standard in cui le particelle non sono più le entità di riferimento ma sono solo specificazione dello stato dei campi e, come tali, posso- 
no venire create o distrutte in relazione a processi di scambio di energia e momento tra campi.

Abbiamo così una Teoria Quantistica dei Campi e proprio in relazione a questa un'ultima circostanza interessa la giustificazione del sistema periodico. L'esistenza di uno spin non è ovviamente una caratteristica esclusiva degli elettroni. Ogni particella è caratterizzata oltre che dal valore della sua massa da quello del suo spin. Si parla di valore dello spin $s$ con riferimento al massimo valore della sua componente nella direzione di riferimento espressa sempre in unità di $\frac{h}{2 \pi}$. Secondo una teoria generale del momento angolare la componente $m_{s}$ nelle stesse unità può assumere tutti i valori che differiscono per interi tra $-s$ e $+s$ e, coerentemente, $s$ può assumere solo valori interi o semidispari. Vi sono in effetti particelle senza spin, con $s=0$ e nessuno stato interno, come i cosiddetti mesoni scalari e pseudoscalari; con $s=\frac{1}{2}$ e due stati possibili $m_{s}= \pm \frac{1}{2}$ come gli elettroni, i protoni, i neutroni; $s=1$ e tre stati interni possibili, $m_{s}=-1,0,1$, come i mesoni vettoriali o gli stessi fotoni. ${ }^{6}$

Nella teoria non relativistica si può tener conto dei gradi di libertà di spin in maniera del tutto analoga a quanto fatto nel caso dell'elettrone, semplicemente introducendo nell'espressione della funzione d'onda un ulteriore coordinata $\omega$, che caso per caso vari su un diverso insieme di valori discreti. Nella teoria relativistica invece a seconda del valore di $s$ la funzione d'onda ha diverse proprietà di trasformazione sotto trasformazioni di Lorentz (scalare, spinoriale, vettoriale, ecc.) e obbedisce ad una diversa equazione. In relazione a questo fatto esiste un importante teorema, dovuto al solito Pauli, detto di connessione tra spin e statistica, secondo il quale, se si vogliono soddisfare alcune importanti proprietà di carattere generale, $i$ campi corrispondenti a spin intero $0,1, \ldots$ devono essere quantizzati secondo le regole che danno luogo a funzioni d'onda completamente simmetriche, quelli corrispondenti a spin semidispari, $\frac{1}{2}, \frac{3}{2}, \ldots$ secondo quelle che danno luogo a funzioni completamente antisimmetriche.

6 Per proprietà generali delle rappresentazioni del gruppo delle trasformazioni di Lorentz le particelle di massa nulla, come il fotone, hanno in ogni caso solo due orientazioni possibili per lo spin, corrispondenti ai valori $\pm_{S}$ di $m_{S}$. Nel csp del fotone i due stati interni si possono presentare anche come due stati di polarizzazione. 


\section{ConClusioni}

Come preannunciato nell'introduzione, in questo contributo ho voluto molto brevemente ripercorrere la via attraverso cui i chimici del XIX sono arrivati, su base puramente empirica, alla costruzione della Tavola Periodica degli elementi; una acquisizione di fondamentale importanza per la disciplina, per l'ordine che ha permesso di stabilire nel settore e il quadro, si può dire, anche di grande bellezza che ha offerto. Ho poi cercato di mostrare il ruolo che ha avuto invece la Fisica nel secolo successivo, nel farne comprenderne il significato profondo e nel fornire una teoria soddisfacente del legame chimico che ha permesso di aprirsi tra l'altro a ad applicazioni e prospettive prima impensabili. Ho ricordato come una sempre più completa giustificazione della tavola abbia seguito il progressivo sviluppo di una Fisica Atomica con un primo fondamentale apporto della cosiddetta vecchia Teoria dei Quanti, la piena coerenza raggiunta con la nuova Meccanica Quantistica e infine un importante contributo anche della Teoria Quantistica dei Campi attraverso il teorema di connessione tra spin e statistica.

\section{BIBLIOGRAFIA}

Enciclopedia della Scienza e della Tecnica, voci: Chimica, Chimica inorganica, Sistema periodico, Legame chimico, Mondadori, Milano 1963.

D. Ter HaAr, The Old Quantum Mechanics, Pergamon Press, Oxford 1967.

G. Ludwig, Wave Mechanics, Pergamon press, Oxford 1968.

M. Born, Fisica Atomica, Boringhieri, Torino 1968.

W. HeISEnBERG, Iprincipi fisici della Teoria dei Quanti, Einaudi, Torino 1953.

G. NATTA e M. FARINA, Stereochimica, molecole in 3D, Mondadori, Milano, 1968.

P. Caldirola, R Cirelli e G.M. ProsPeri, Introduzione alla Fisica Teorica, UTET, Torino, 1982.

B. H. Brandsen and C. J. Joachain, Physics of Atoms and Molecules, second edition, Pearson Education, Harlow England, 2003.

C. A. Coulson and J. T. Lewis, Chemical Binding, su Quantum Theory, D. R. BATES, ed., vol, II Academic Press, New York, 1982.

S. WeInBERG, The Quantum Theory of Fields, vol. I, Candbidge University Press. 